\title{
Resultados de técnicas laparoscópicas para herniorrafia inguinal: uma revisão integrativa
}

\author{
Results of laparoscopic techniques for inguinal herniorraphy: an integrative review
}

Resultados de técnicas laparoscópicas para herniorrafía inguinal: una revisión integrativa

Izabella Sena de Oliveira ${ }^{1 *}$, Amyr Abdala Gomes ${ }^{1}$, Bruna Paiva de França ${ }^{1}$, Eduarda Luz Barbosa Alarcão ${ }^{1}$, Priscila Chaves Cruz ${ }^{1}$, Stephany Benelli Canal ${ }^{1}$, Alberto Vilar Trindade ${ }^{1}$.

\section{RESUMO}

Objetivo: Comparar a Técnica Laparoscópica Totalmente Extraperitoneal (TEP) e a Técnica Laparoscópica Transabdominal Pré-peritoneal (TAPP) para a correção de hérnias inguinais. Métodos: Buscou-se avaliar as melhores circunstâncias para a utilização de cada uma. Realizada revisão integrativa nos bancos de dados PubMed, SciELO, LILACS e UpToDate, com os descritores DeCS: "inguinal hernia" AND "laparoscopy" AND "TEP" AND "TAPP", e seus equivalentes em português. Resultados: As diferenças entre os resultados finais e as complicações não apresentaram grande divergência estatística. A TEP demonstrou redução da dor pósoperatória e menor tempo de internação hospitalar, mas apresenta curva de aprendizado mais longa e espaço de abordagem restrito, fatores que a tornam mais difícil. Apresentou também risco aumentado de lesões vasculares e maior incidência de complicações pós-operatórias. Já a TAPP, demonstrou facilidade no aprendizado, porém evoluiu com maiores riscos de aderências intestinais à tela, de lesões viscerais durante a entrada transabdominal e de dor no pós-operatório. Considerações finais: Não há diferença significativa entre as técnicas quanto a incidência de complicações pós-operatórias, a recorrência de reabordagem e o impacto na qualidade de vida dos pacientes. A decisão da melhor abordagem deverá ficar a critério do cirurgião e do serviço.

Palavras-chave: Hérnia inguinal, Hernioplastia, Técnica laparoscópica totalmente extraperitoneal, Técnica laparoscópica transabdominal pré-peritoneal.

\begin{abstract}
Objective: To compare a Totally Extraperitoneal Laparoscopic Technique (TEP) and a Transabdominal Preperitoneal Laparoscopic Technique (TAPP) for an inguinal hernia repair. Methods: It was sought to assess the best conditions for using each. An integrative review was carried out in the PubMed, SciELO, LILACS and UpToDate databases, with the DeCS descriptors: " inguinal hernia " AND " laparoscopy " AND " TEP " AND " TAPP ", and their respective in portuguese. Results: The differences between the final results and the complications did not show statistical divergence. TEP has shown reduced postoperative pain and shorter hospital stay, but it has a longer learning curve and restricted space for approach, factors that make it more difficult. There was also an increased risk of vascular injuries and a higher incidence of postoperative complications. TAPP, on the other hand, demonstrated ease in learning, but evolved with greater risks of intestinal adhesions to the mesh, visceral injuries during transabdominal entry, and postoperative pain. Final considerations: There is no difference between the techniques regarding the incidence of postoperative complications, recurrence of re-approach and the impact on the patient's quality of life. The decision on the best approach should be left to the surgeon and the service.
\end{abstract}

Keywords: Inguinal hernia, Hernioplasty, Totally extraperitoneal laparoscopic technique, Transabdominal preperitoneal laparoscopic technique.

${ }^{1}$ Centro Universitário de Brasília (CEUB), Brasília - DF. *E-mail: izabella.sena@sempreceub.com 


\section{RESUMEN}

Objetivos: Comparar la Técnica Laparoscópica Totalmente Extraperitoneal (TEP) y la Técnica Laparoscópica Transabdominal Preperitoneal (TAPP) para la corrección de hernias inguinales. Métodos: Se buscó evaluar las mejores circunstancias para usar cada una. Se realizó una revisión integradora en las bases de datos PubMed, SciELO, LILACS y UpToDate, con los descriptores DeCS: "hernia inguinal" Y "laparoscopia" Y "TEP" $Y$ "TAPP", y sus equivalentes en portugués. Resultados: Las diferencias entre los resultados finales y las complicaciones no mostraron gran divergencia estadística. La TEP demostró reducción del dolor posoperatorio y estancia hospitalaria más corta, pero tiene una curva de aprendizaje más larga y un espacio restringido para el abordaje, factores que lo hacen más difícil. También presentó mayor riesgo de lesiones vasculares y mayor incidencia de complicaciones posoperatorias. TAPP, por su parte, demostró una facilidad de aprendizaje, pero evolucionó con mayores riesgos de adherencias intestinales a la malla, lesiones viscerales durante la entrada transabdominal y dolor posoperatorio. Consideraciones finales: No existe diferencia significativa entre las técnicas en cuanto a la incidencia de complicaciones postoperatorias, la recurrencia del nuevo abordaje y el impacto en la calidad de vida de los pacientes. La decisión sobre el mejor enfoque debe dejarse al cirujano y al servicio.

Palabras clave: Hernia inguinal, Hernioplastia, Técnica laparoscópica totalmente extraperitoneal, Técnica laparoscópica transabdominal preperitoneal.

\section{INTRODUÇÃO}

As hérnias são caracterizadas pela protrusão de uma estrutura anatômica através de uma estrutura que a continha, como a parede abdominal (VACCA VM, 2017). A hérnia inguinal, por sua vez, decorre do enfraquecimento dos tecidos que compõem o canal inguinal. A formação de uma hérnia é denominada como herniação, e existem duas maneiras nas quais pode ocorrer, definindo a hérnia como hérnia inguinal direta ou hérnia inguinal indireta (VACCA VM, 2017; ARRUDA WMQ, et al., 2019).

A correção da hérnia inguinal - denominada herniorrafia - é considerada o procedimento da cirurgia geral mais realizado no mundo, com uma média de 20 milhões de cirurgias todos os anos (BANSAL VK, et al., 2017; ŁOMNICKI J, et al., 2018; VARCUS F, et al., 2016). A prevalência de hérnias na população mundial representa cerca de $1,7 \%$ para todas as idades, enquanto nos indivíduos acima dos 45 anos de idade observase um aumento para 4\% (ARRUDA WMQ, et al., 2019).

As hérnias inguinais compõem $75 \%$ de todas as hérnias de parede abdominal, cujo risco de ocorrência nas mulheres é de $3 \%$ e nos homens, de $27 \%$ ao longo da vida (ARRUDA WMQ, et al., 2019; AIOLFI A, et al., 2019). Homens acima de 75 anos são o grupo com maior incidência, sendo que 200 a cada 10.000 são acometidos por ano, enquanto homens com idade entre 16 e 24 anos apresentam uma incidência de 11 para cada 10.000 por ano (ARRUDA WMQ, et al., 2019).

Devido sua alta incidência, diversas técnicas para seu reparo foram desenvolvidas. Contudo, a cirurgia que utiliza a técnica de Lichtenstein continua sendo muito utilizada no mundo (AIOLFI A, et al., 2019; LYU Y, et al., 2020). Com a introdução da laparoscopia na cirurgia, novos métodos foram desenvolvidos, como a técnica laparoscópica totalmente extraperitoneal (TEP) e a técnica laparoscópica transabdominal préperitoneal (TAPP), que se tornaram as mais utilizadas (LIU Y, et al., 2020).

Várias pesquisas comparando as técnicas de cirurgia aberta e laparoscópica foram realizadas e demonstraram que as laparoscópicas apresentavam tempo de recuperação menor, dores pós-cirúrgicas de menor intensidade e menos complicações pós-operatórias (CHEN L, et al., 2019; BANSAL VK, et al., 2013). Entretanto, ainda não foi determinada uma superioridade entre as técnicas TEP e TAPP. A técnica TEP apresenta a vantagem de não invadir a cavidade peritoneal, porém apresenta maior dificuldade devido à restrição de espaço durante o procedimento. Por outro lado, a técnica TAPP penetra a cavidade peritoneal, mas é de mais fácil realização e com mais espaço para o procedimento (BANSAL VK, et al., 2013). 
Assim, esse estudo tem a finalidade de revisar a literatura para comparar a TEP com a TAPP na realização de herniorrafias. Ademais, pretende determinar as melhores circunstâncias para a utilização de cada técnica e comparar suas similaridades e diferenças no tratamento dessa doença.

\section{MÉTODOS}

Para a revisão integrativa foram utilizadas bases de dados eletrônicos para a pesquisa de artigos e publicações que se relacionavam ao tratamento de hérnias inguinais, utilizando as técnicas laparoscópicas totalmente extraperitoneal (TEP) ou transabdominal pré-peritoneal (TAPP). As bases de dados eletrônicas utilizadas foram Medline (PubMed), SciELO, LILACS e UpToDate, selecionando artigos datados nos últimos 5 anos, entre 2015 e 2021. Os descritores DeCS utilizados no trabalho foram "Inguinal Hernia" AND "Laparoscopy" AND "TEP" AND "TAPP" e seus correspondentes em português.

Os trabalhos agrupados passaram por um processo de seleção, tendo como critério de inclusão estudos sobre correções de hérnias inguinais utilizando as técnicas laparoscópicas TEP ou TAPP. Não houve restrição quanto ao idioma dos artigos.

Figura 1 - Fluxograma da busca eletrônica dos artigos.

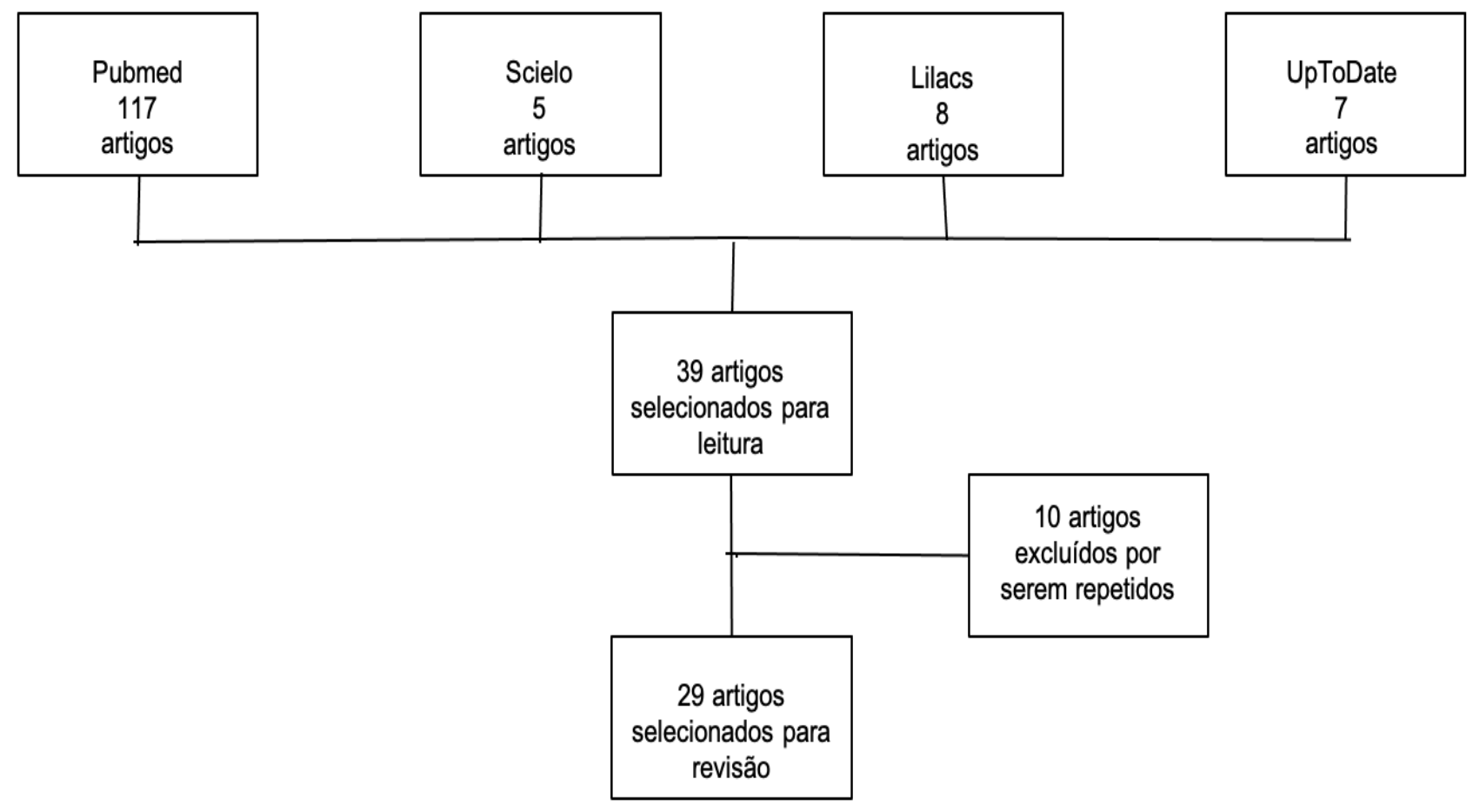

Fonte: Oliveira IS, et al., 2021.

\section{RESULTADOS E DISCUSSÃO}

As técnicas laparoscópica TAPP e TEP, empregadas para a correção da hérnia inguinal, são amplamente utilizadas, e, cada uma dessas técnicas possuem suas características. Muitas vezes, devido à semelhança entre ambas, os especialistas da área apresentam uma dificuldade na aplicação das mesmas, sendo de extrema importância a diferenciação entre seus pontos positivos e negativos. Visando a compilação desses dados, realizou-se a tabela abaixo, na qual apresenta os principais achados de cada estudo encontrado, sendo descrito também o título, autores e ano de publicação de cada estudo. 
Quadro 1 - Apresentação dos resultados dos artigos selecionados na revisão.

\begin{tabular}{|c|c|c|c|}
\hline TITULO & AUTORES/ ANO & TIPO DE ESTUDO & PRINCIPAIS RESULTADOS/ CONCLUSŌES \\
\hline $\begin{array}{l}\text { Laparoscopic totally extraperitoneal vs robotic } \\
\text { transabdominal preperitoneal inguinal hernia repair: } \\
\text { Assessment of short-and long-term outcomes. }\end{array}$ & $\begin{array}{l}\text { AGHAYEVA A, et al., } \\
2020\end{array}$ & Estudo retrospectivo & $\begin{array}{l}\text { O estudo apresentou desfechos pós-operatórios semelhantes quando } \\
\text { comparados os resultados a longo prazo e taxas de recorrência entre a } \\
\text { correção de hérnias inguinais. Evidenciou que o tempo operatório foi } \\
\text { significativamente maior para a técnica de TAPP quando em comparação à } \\
\text { TEP. Demonstrou que na TEP há redução da dor pós operatória, sobretudo } \\
\text { quando é a primeira abordagem da hérnia inguinal e que na TAPP há uma } \\
\text { maior facilidade de aprendizado, contudo há um maior risco de lesões } \\
\text { viscerais. }\end{array}$ \\
\hline $\begin{array}{l}\text { Primary inguinal hernia: systematic review and } \\
\text { Bayesian network meta-analysis comparing open, } \\
\text { laparoscopic transabdominal preperitoneal, totally } \\
\text { extraperitoneal, and robotic preperitoneal repair. }\end{array}$ & AIOLFI A, et al., 2019 & $\begin{array}{l}\text { Revisão sistemática e } \\
\text { metanálise }\end{array}$ & $\begin{array}{l}\text { Demonstrou que as técnicas analisadas (TAPP, TEP e aberta) possuíam } \\
\text { semelhança a curto prazo e que a técnica a ser realizada deveria ser baseada } \\
\text { no conhecimento individual do cirurgião e nas características de cada } \\
\text { paciente. }\end{array}$ \\
\hline $\begin{array}{l}\text { Comparação entre as técnicas laparoscópica } \\
\text { totalmente extraperitoneal (TEP) e transabdominal } \\
\text { pré-peritoneal (TAPP) na hernioplastia inguinal: } \\
\text { síntese de evidências clínicas. }\end{array}$ & $\begin{array}{c}\text { ARRUDA WMQ, et al., } \\
2019\end{array}$ & Revisão bibliográfica & $\begin{array}{l}\text { O estudo não observou diferenças entre as técnicas TEP e TAPP, } \\
\text { apresentando ambas como opções cirúrgicas viáveis para a correção das } \\
\text { hérnias inguinais. Em adendo, sugere que seja considerado previamente o } \\
\text { tamanho do defeito da hérnia e os riscos de lesão de cada técnica. }\end{array}$ \\
\hline $\begin{array}{l}\text { A prospective randomized comparison of sexual } \\
\text { function and semen analysis following laparoscopic } \\
\text { totally extraperitoneal (TEP) and transabdominal pre- } \\
\text { peritoneal (TAPP) inguinal hernia repair. }\end{array}$ & ASURI K, et al., 2021 & $\begin{array}{l}\text { Ensaio clínico } \\
\text { randomizado }\end{array}$ & $\begin{array}{l}\text { Ambas as técnicas TEP e TAPP demonstraram comparável melhora em } \\
\text { relação à função sexual e ao efeito sob o sêmen em pacientes com hérnia } \\
\text { inguinal. }\end{array}$ \\
\hline $\begin{array}{l}\text { Guidelines for laparoscopic (TAPP) and endoscopic } \\
\text { (TEP) treatment of inguinal hernia [International } \\
\text { Endohernia Society (IEHS)]. }\end{array}$ & $\begin{array}{l}\text { BITTNER R, et al., } \\
2011\end{array}$ & $\begin{array}{l}\text { Guideline (Revisão } \\
\text { sistemática) }\end{array}$ & $\begin{array}{l}\text { Realizada visando determinar os aspectos, indicações, precauções e } \\
\text { possíveis riscos das técnicas TAPP e TEP. }\end{array}$ \\
\hline $\begin{array}{l}\text { Update of guidelines on laparoscopic (TAPP) and } \\
\text { endoscopic (TEP) treatment of inguinal hernia } \\
\text { (INternational Endohernia Society) }\end{array}$ & $\begin{array}{c}\text { BITTNER R, et al., } \\
2014\end{array}$ & $\begin{array}{l}\text { Guideline (Revisão } \\
\text { sistemática) }\end{array}$ & $\begin{array}{l}\text { Foi reportado um índice de dor pós-operatória significativamente maior em } \\
\text { pacientes que passaram pelo método TAPP, foram avaliados períodos entre } \\
6 \text { horas e } 90 \text { dias, sendo a TAPP persistente com uma maior sensação } \\
\text { dolorosa em todas as ocasiões de avaliação. Foi reportado também a } \\
\text { necessidade de maiores doses de analgésicos para lidar com essa dor após } \\
\text { a TAPP. Houve um maior índice de seromas em pacientes que passaram pela } \\
\text { TEP, mas por outro lado houve maior incidência de edema de bolsa escrotal } \\
\text { em pacientes que passaram pela TAPP. Contudo não foram encontradas } \\
\text { diferenças significativas entre esses dois métodos. }\end{array}$ \\
\hline
\end{tabular}




\begin{tabular}{|c|c|c|c|}
\hline TITULO & AUTORES/ ANO & TIPO DE ESTUDO & PRINCIPAIS RESULTADOS/ CONCLUSŌES \\
\hline Inguinal hernia repair: current surgical techniques & $\begin{array}{l}\text { BITTNER R e } \\
\text { SCHWARZ J, } 2011\end{array}$ & Revisão Bibliográfica & $\begin{array}{l}\text { Sobre a técnica TAPP foi relatada maior incidência de menor tempo de } \\
\text { internação pós-operatório, número de lesões viscerais e menor número de dor } \\
\text { aguda pós-operatória. Já no método TEP foram reportados maior número de } \\
\text { complicações vasculares. }\end{array}$ \\
\hline $\begin{array}{l}\text { Primary unilateral not complicated inguinal hernia: our } \\
\text { choice of TAPP, why, results and review of literature }\end{array}$ & $\begin{array}{l}\text { BITTNER R, } \\
\text { SCHWARZ J, } 2019\end{array}$ & Revisão Sistemática & $\begin{array}{l}\text { O resultado final revela que não há evidência suficiente para qualificar um } \\
\text { método como melhor do que o outro. Esse estudo realça o menor grau de dor } \\
\text { pós-operatória e o menor tempo de internação pós-operatória e de melhora } \\
\text { do paciente observados no método TAPP. Contudo, ambos os métodos ainda } \\
\text { são recomendados, não possuindo diferenças significativas entre eles. Dessa } \\
\text { forma o método mais recomendado é aquele o qual o cirurgião está mais } \\
\text { confortável e capacitado em realizar. }\end{array}$ \\
\hline $\begin{array}{l}\text { Overview of treatment for inguinal and femoral hernia } \\
\text { in adults. }\end{array}$ & BROOKS D, 2020 & - & - \\
\hline $\begin{array}{l}\text { Effects of transabdominal preperitoneal and totally } \\
\text { extraperitoneal inguinal hernia repair: an update } \\
\text { systematic review and meta-analysis of randomized } \\
\text { controlled trials }\end{array}$ & CHEN L, et al, 2018 & Metanálise & $\begin{array}{l}\text { Foram analisados } 1516 \text { pacientes divididos entre grupos TAPP e TEP. } \\
\text { Diferentemente de outros estudos, foi averiguado que o método TEP está } \\
\text { correlacionado com menor tempo de recuperação pós-operatório, assim como } \\
\text { menor grau de dor a curto-prazo em casos primários, porém maior tempo de } \\
\text { cirurgia em casos de hérnia recorrente. Enquanto o método TAPP demonstrou } \\
\text { menores tempos de cirurgia. }\end{array}$ \\
\hline $\begin{array}{l}\text { Experience and results of laparoscopic inguinal } \\
\text { herniorrhaphy }\end{array}$ & $\begin{array}{l}\text { HAZBÓN HR, LÓPEZ- } \\
\text { ATEHORTUA DF, } 2017\end{array}$ & Estudo retrospectivo & $\begin{array}{l}\text { Dentre os } 100 \text { casos de hérnia inguinal operados laparoscopicamente, } 92 \\
\text { foram monitorados no pós-operatório. Entre eles, três }(3,2 \%) \text { necessitaram de } \\
\text { conversão, três ( } 3,2 \%) \text { tiveram recorrência da hérnia e seis }(6,4 \%) \\
\text { apresentaram dor crônica. A herniorrafia laparoscópica pela técnica de TAPP } \\
\text { é um procedimento mais seguro. A eficácia e as complicações demonstraram } \\
\text { ser semelhantes às técnicas aberta e TEP. }\end{array}$ \\
\hline $\begin{array}{l}\text { Safety of two common laparoscopic inguinal } \\
\text { herniorrhaphy approaches: an updated systematic } \\
\text { review with meta-analysis of randomized clinical trials }\end{array}$ & HUNG TY, et al, 2007 & Metanálise & $\begin{array}{l}\text { TEP apresenta uma maior taxa de seroma quando comparada ao TAPP, } \\
\text { entretanto, apresenta uma menor taxa de edema escrotal no pós-operatório } \\
\text { imediato e após } 1 \text { semana. Assim, as duas técnicas têm suas vantagens e a } \\
\text { decisão compartilhada quanto a técnica laparoscópica escolhida ainda é } \\
\text { necessária. }\end{array}$ \\
\hline $\begin{array}{l}\text { Laparoscopic totally extraperitoneal (TEP) } \\
\text { hernioplasty using two trocars: anatomical landmarks } \\
\text { and surgical technique }\end{array}$ & $\begin{array}{l}\text { IUAMOTO LR, et al., } \\
2015\end{array}$ & Artigo original & $\begin{array}{l}\text { A realização da herniorrafia com abordagem TEP utilizando apenas dois } \\
\text { trocárteres é uma alternativa viável, com melhora dos resultados estéticos, } \\
\text { financeiros e menor dor no pós-operatório. }\end{array}$ \\
\hline
\end{tabular}




\begin{tabular}{|c|c|c|c|}
\hline TÍTULO & AUTORES/ ANO & TIPO DE ESTUDO & PRINCIPAIS RESULTADOS/ CONCLUSÖES \\
\hline $\begin{array}{l}\text { TEP versus TAPP: comparison of the perioperative } \\
\text { outcome in } 17,587 \text { patients with a primary unilateral } \\
\text { inguinal hernia }\end{array}$ & $\begin{array}{l}\text { KÖCKERLING F, et al., } \\
2015\end{array}$ & Estudo prospectivo & $\begin{array}{l}\text { Não houve diferença significativa entre as taxas de complicações intra- } \\
\text { operatórias pós-operatórias e taxa de reoperação devido à complicações } \\
\text { quanto às técnicas TAPP e TEP. A maior taxa de complicações pós- } \\
\text { operatórias foi para TAPP, mas essa taxa é parcialmente explicada pela idade } \\
\text { avançada, maior tamanho do defeito e mais hérnias escrotais. }\end{array}$ \\
\hline $\begin{array}{l}\text { Current treatment of the inguinal hernia: the role of the } \\
\text { totally extraperitoneal (TEP) hernia repair }\end{array}$ & $\begin{array}{l}\text { ŁOMNICKI J, et al., } \\
\qquad 2018\end{array}$ & Revisão bibliográfica & $\begin{array}{l}\text { O método TEP parece ser equivalente quando comparado com o método } \\
\text { TAPP. Os benefícios de uma técnica com abordagem pré-peritoneal são os } \\
\text { riscos reduzidos de lesão de órgãos abdominais. A cirurgia TEP continua } \\
\text { sendo um dos principais reparos de hérnia inguinal. A escolha de uma técnica } \\
\text { cirúrgica espećfica depende das preferências do cirurgiẫo e das } \\
\text { características do paciente. Sem dúvida, o método TEP tem suas vantagens } \\
\text { e é recomendado pelas diretrizes da Sociedade de Hérnia Europeia em } \\
\text { hérnias bilaterais e em hérnias recorrentes. }\end{array}$ \\
\hline $\begin{array}{l}\text { Comparison of endoscopic surgery and Lichtenstein } \\
\text { repair for treatment of inguinal hernias }\end{array}$ & LYU Y, et al., 2020 & Metanálise & $\begin{array}{l}\text { Não houve diferenças significativas entre os } 3 \text { procedimentos quanto a } \\
\text { recorrência de hérnia, dor crônica, hematoma, seroma e internações. O } \\
\text { método Lichtenstein teve um tempo de operação mais curto do que TAPP e } \\
\text { TEP: } 12 \text { versus } 18 \text { minutos, respectivamente, mas foi associado com mais } \\
\text { infecções de feridas do que TEP. A metanálise sugere que TAPP e TEP } \\
\text { exigem menos dias de retorno ao trabalho. }\end{array}$ \\
\hline $\begin{array}{l}\text { Laparoscopic Inguinal Hernia Repair: Transabdominal } \\
\text { Preperitoneal or Totally Extraperitoneal? Results of a } \\
\text { 14-year Prospective Study }\end{array}$ & PATEL V, et al., 2020 & Estudo prospectivo & $\begin{array}{l}\text { 49,5\% dos reparos pela técnica TAPP foram hérnias recorrentes e } 95,5 \% \text { pela } \\
\text { técnica TEP foram hérnias bilaterais. Os pacientes TAPP eram } \\
\text { significativamente mais velhos do que os pacientes TEP }(60,65 \text { versus } 55,60 \text {, } \\
\text { p<0,01). As cirurgias TAPP unilaterais tiveram tempo operacional } \\
\text { significativamente menor do que as TEP unilaterais }(50,94 \text { versus } 65,71 \\
\text { minutos, } p=0,01) \text {. Não houve diferença significativa na taxa de complicação } \\
\text { entre os reparos TAPP e TEP }(6,84 \% \text { versus } 7,38 \%, p=0,87) \text {. A recorrência } \\
\text { pelo método TAPP possuiu uma taxa significativamente maior do que o } \\
\text { método TEP }(3,16 \% \text { versus } 0,61 \%, p=0,02) \text { em geral. }\end{array}$ \\
\hline $\begin{array}{l}\text { Laparoscopic inguinal and femoral hernia repair in } \\
\text { adults }\end{array}$ & $\begin{array}{l}\text { SAROSI GA e BEN- } \\
\text { DAVID K., } 2015\end{array}$ & Revisão bibliográfica & $\begin{array}{l}\text { O procedimento TEP é tecnicamente mais desafiador. Para a maioria dos } \\
\text { pacientes do sexo masculino, sugerimos a abordagem TEP, desde que o } \\
\text { cirurgião tenha experiência. Para pacientes em que a técnica de TEP não é é } \\
\text { adequada (hérnia grande, cirurgia prévia de linha média inferior) ou falha } \\
\text { devido à incapacidade de desenvolver o espaço pré-peritoneal, a conversão } \\
\text { para uma abordagem TAPP pode ser realizada. Em comparação com o TAPP, } \\
\text { o TEP está associado a uma maior taxa de recorrência, mas a uma menor } \\
\text { taxa de complicaçôes. Ambas as técnicas laparoscópicas estão associadas } \\
\text { com menos dor crônica e dormência do que no manejo por laparotomia. }\end{array}$ \\
\hline
\end{tabular}




\begin{tabular}{|c|c|c|c|}
\hline TÍTULO & AUTORES/ ANO & TIPO DE ESTUDO & PRINCIPAIS RESULTADOS/ CONCLUSŌES \\
\hline 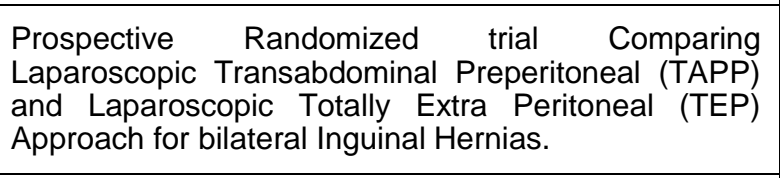 & $\begin{array}{l}\text { SHARMA D, et al., } \\
\qquad 2015\end{array}$ & $\begin{array}{l}\text { Ensaio clínico } \\
\text { randomizado }\end{array}$ & $\begin{array}{l}\text { Os resultados das duas técnicas operatórias foram similares. As principais } \\
\text { diferenças encontradas foram: o tempo médio de operação aumentado e os } \\
\text { escores de dor pós- operatória imediata elevados no método TEP. Ademais, } \\
\text { algumas complicações, como o enfisema subcutâneo apresentou-se mais } \\
\text { prevalente na técnica TEP. }\end{array}$ \\
\hline $\begin{array}{l}\text { Comparison of the effect of mesh-plug, Lichtenstein, } \\
\text { Transabdominal Preperitoneal, and Totally } \\
\text { Extraperitoneal Hernia Repair. }\end{array}$ & SHI TH, et al., 2020 & Metanálise & $\begin{array}{l}\text { O estudo mostrou que não há diferenças significantes entre as duas técncias } \\
\text { no tempo de operação, surgimento de seroma, infecções ou recidiva. }\end{array}$ \\
\hline $\begin{array}{l}\text { Experiencia Inicial en el Tratamiento Laparoscópico de } \\
\text { la Hernia Inguinal en la II Cátedra de Clinica } \\
\text { Quirúrgica, Hospital de Clínicas FCM-UNA. }\end{array}$ & $\begin{array}{l}\text { TORRES OC, et al., } \\
2016\end{array}$ & Estudo retrospectivo & $\begin{array}{l}\text { Dos } 25 \text { pacientes avaliados, } 5 \text { foram operados com a técnica TEP e } 20 \text { a } \\
\text { TAPP. Dentre esses } 20 \text { indivíduos, um apresentou seroma, um infeç̧ão de } \\
\text { sítio cirúrgico e um inguinodinia. }\end{array}$ \\
\hline $\begin{array}{l}\text { TAAP vs. TEP in Inguinal Hernia Repair - What is the } \\
\text { Evidence? A Single Center Experience }\end{array}$ & TULIN A, et al., 2019 & Estudo retrospectivo & $\begin{array}{l}\text { As vantagens e desvantagens das duas técnicas variam: } \\
\text { A TEP requer equipamento especializado e é praticada em hérnias laterais, } \\
\text { há um aumento do risco de sangramento ao dissecar o espaço pré-peritoneal. } \\
\text { A técnica TAAP vem com a vantagem de maior espaço de trabalho para } \\
\text { manobrar os instrumentos laparoscópicos, mas apresenta um risco } \\
\text { aumentado de ferir órgãos abdominais. Devido ao uso de } \\
\text { mais grampos de fixação na TAAP para cobrir a malha com } \\
\text { peritônio, os custos săo aumentados. }\end{array}$ \\
\hline Inguinal Hernia: A battle of the Bulge & VACCA VM, 2017 & Livro & \\
\hline $\begin{array}{l}\text { Consensus on International Guidelines for } \\
\text { Management of Groin Hernias }\end{array}$ & $\begin{array}{l}\text { VAN VEENENDAAL N, } \\
\text { et al., } \\
2020\end{array}$ & Editorial & $\begin{array}{l}822 \text { cirurgiões votaram na preferência entre as técnicas cirúrgicas para reparo } \\
\text { de hénia inguinal. Desses, a preferência de } 30,9 \% \text { dos participantes foi pelo } \\
\text { reparo de Lichtenstein, } 29,6 \% \text { preferem o reparo por TEP e } 24,3 \% \text { preferem } \\
\text { a técnica TAPP. }\end{array}$ \\
\hline $\begin{array}{l}\text { Laparoscopic Repair of Inguinal Hernia TEP versus } \\
\text { TAPP }\end{array}$ & $\begin{array}{l}\text { VARCUS F, et al., } \\
2016\end{array}$ & $\begin{array}{l}\text { Estudo retrospectivo } \\
\text { comparativo }\end{array}$ & $\begin{array}{l}\text { A média de tempo de operação com TEP foi maior do que com TAPP (74 } \\
\text { versus } 69 \text { minutos). A ténica de TEP não apresentou sangramente } \\
\text { intraoperátorio nem lesão de visceras, já a TAPP apresentou } 2 \text { e } 1 \text { episódios, } \\
\text { respectivamente. Não houve recorrência de hérnia em dois anos de } \\
\text { acompanhamento dos } 36 \\
(81,81 \%) \text { pacientes com TEP e } 34(73,91 \%) \text { com TAPP. Não foi registrada } \\
\text { mortalidade em } 30 \text { dias nos pacientes de ambas técnicas. }\end{array}$ \\
\hline $\begin{array}{l}\text { Transabdominal Preperitoneal (TAPP) Versus Totally } \\
\text { Extraperitoneal (TEP) for Laparoscopic Hernia Repair: } \\
\text { A Meta-Analysis }\end{array}$ & $\begin{array}{l}\text { WEI FX, et al., } \\
2015\end{array}$ & Metanálise & $\begin{array}{l}\text { Não houve diferença significativa em termos de recorrência de hérnia, escores } \\
\text { de dor, tempo de operação, tempo para retornar às atividades habituais, } \\
\text { permanência no hospital, complicações totais e custo entre os } 2 \text { grupos. A } \\
\text { análise de subgrupo revelou que os escores de dor seriam afetados por } \\
\text { muitos fatores clínicos, o tempo de operação foi determinado principalmente } \\
\text { pelo estado e pela experiência do cirurgião. Com base nas evidências atuais, } \\
\text { o TEP como um procedimento laparoscópico modificado e mais complexo do } \\
\text { que o TAPP, não levou a uma diferença significativa nos aspectos de } \\
\text { resultados clínicos e complicações. Portanto, o estudo recomenda } \\
\text { primeiramente o TAPP para correção de hérnia laparoscópica, especialmente } \\
\text { para cirurgiões não especialistas. }\end{array}$ \\
\hline
\end{tabular}


O tratamento definitivo de qualquer tipo de hérnia, incluindo a inguinal, é a abordagem cirúrgica. As técnicas laparoscópicas têm ganhado maior visibilidade, tendo em vista o menor tempo de internação e dor no pós-operatório, bem como a recuperação acelerada e retorno precoce ao trabalho (LYU Y, et al., 2020; WEI FX, et al., 2015; IUAMOTO LR, et al., 2015).

Nesse contexto, a realização da herniorrafia pode ser necessária de maneira urgente ou não, a depender do tipo de hérnia e de suas complicações. Dentre as técnicas laparoscópicas, a TEP e a TAPP são as mais utilizadas (BROOKS DC, 2020). No entanto, a escolha do procedimento é uma decisão particular e individualizada, visto que nenhum método será adequado para todos os tipos de hérnia. Nessa escolha, devem ser valorizadas a taxa de complicação baixa, as particularidades do paciente, o menor tempo de recuperação, o custo-efetividade e a capacidade e experiência do cirurgião (ŁOMNICKI J, et al., 2018; BITTNER R e SCHWARZ J, 2019).

A TAPP e a TEP são bem aceitas na atualidade, mas ainda há um déficit de estudos que comparam a segurança e eficácia entre elas. A TEP foi desenvolvida a partir da TAPP, abordagem original, a fim de diminuir algumas complicações associadas à mesma. Entretanto, apresenta espaço de trabalho mais limitado, tornando-se mais complexa (SAROSI GA e BEN-DAVID K, 2015). Dessa forma, apesar de ser a técnica de escolha de muitos cirurgiões, nem todos têm a capacidade de realizá-la bem tendo em vista a sua curva de aprendizado de 60 a 70 procedimentos (IUAMOTO LR, et al., 2015)

Para cirurgiões que apresentam excelência na realização das duas técnicas, recomenda-se a escolha da TEP para a maioria dos pacientes do sexo masculino. Além disso, também é indicada para pacientes que apresentam hérnia inguinal primária e bilateral, não sendo tão indicada nos casos de hérnia recorrente devido a formação de tecido cicatricial entre as camadas da parede abdominal, que torna a criação de um espaço pré-peritoneal mais difícil. Em pacientes que apresentam aderências intra-abdominais também é preferível a TEP, que não adentra esse espaço. Outrossim, evidencia-se que a TEP pode ser realizada sem a necessidade de anestesia geral (BROOKS DC, 2020; SAROSI GA e BEN-DAVID K, 2015; PATEL V, et al., 2020).

Nos pacientes em que a técnica extraperitoneal é falha devido à incapacidade do desenvolvimento do espaço pré-peritoneal, indica-se o uso da TAPP. Essa técnica também é indicada para pacientes do sexo feminino, tendo em vista melhores resultados e menor taxa de recorrência, e pacientes com hérnia inguinal unilateral e recorrente. Além disso, pacientes que tenham realizado uma cirurgia pélvica anterior ou apresentem hérnia oculta, o método de escolha é a TAPP (SAROSI GA e BEN-DAVID K, 2015; PATEL V, et al., 2020). Destaca-se que pacientes com ascite devem evitar a abordagem laparoscópica, em especial a TAPP (BROOKS DC, 2020).

\section{Técnicas}

\section{Técnica laparoscópica totalmente extraperitoneal (TEP)}

A TEP foi inicialmente descrita por McKernan e Laws, em 1993. Essa técnica não invade a cavidade peritoneal, pois o acesso, o reparo da hérnia e o posicionamento da tela ocorrem no espaço extraperionetal, evitando qualquer risco de lesão visceral, infecção e diminuindo a dor por incisão do peritônio parietal. Entretanto, por apresentar espaço restrito, pode falhar, devendo ser convertida para a TAPP (WEI FX, et al., 2015; SAROSI GA e BEN-DAVID K, 2015; PATEL V, et al., 2020; TORRES OC, et al., 2016)

A técnica cirúrgica consiste no estabelecimento de um espaço entre o peritônio e a parede abdominal posterior. Assim, a incisão deve ser feita entre a cicatriz umbilical e o local da hérnia, seguida da incisão e posterior retração lateral do músculo reto abdominal e por fim, o estabelecimento de um espaço pré-peritoneal que pode ser feito com a utilização do balão de Herloon, a câmera laparoscópica ou o próprio dedo (ŁOMNICKI J, et al., 2018) (BITTNER R e SCHWARZ J, 2012).

Após a formação do espaço pré-peritoneal, deve-se inserir um portal pela mesma incisão e em seguida, a câmera através dele. A dissecção deve ser realizada no plano avascular, entre o peritônio e a fáscia transversal, respeitando o limite superior, até a área subumbilical, o limite inferior, até o espaço retropúbico e o limite lateral, até o músculo Psoas e espaço retro-inguinal. Em seguida, dois outros portais são adicionados. 
A localização pode variar dependendo do cirurgião, podendo estar um na linha média entre a cicatriz umbilical e a sínfise púbica e outro próximo à espinha ilíaca ântero-superior - ao lado da hérnia - ou ambos na linha média entre a cicatriz umbilical e a sínfise púbica (ŁOMNICKI J, et al., 2018; SAROSI GA e BEN-DAVID K, 2015; BITTNER R e SCHWARZ J, 2012).

O espaço criado é insuflado através do portal principal e a hérnia é cuidadosamente dissecada na direção cefálica. Hérnias diretas são facilmente reduzidas durante a dissecção e indiretas apresentam maior dificuldade, pois estão aderidas nas estruturas do cordão. Após dissecção, a tela é adicionada e pode ou não ser fixada no ligamento de Cooper, tubérculo púbico, tendão conjunto e músculos reto abdominais (SAROSI GA e BEN-DAVID K, 2015; PATEL V, et al., 2020).

Por fim, os portais são removidos e o espaço pré-peritoneal é esvaziado e fechado com sutura contínua no peritônio parietal (SAROSI GA e BEN-DAVID K, 2015; HAZBÓN HR e LÓPEZ-ATEHORTUA DF, 2017).

\section{Técnica laparoscópica transabdominal pré-peritoneal (TAPP)}

Como a maioria das técnicas laparoscópicas, a TAPP necessita do acesso à cavidade peritoneal, não havendo restrição de espaço, tornando-a menos complexa e permitindo a visualização das regiões inguinais. Entretanto, a invasão da cavidade peritoneal está associada a maiores desvantagens ao paciente (SAROSI GA e BEN-DAVID K, 2015; PATEL V, et al., 2020; TORRES OC, et al., 2016; HAZBÓN HR e LÓPEZATEHORTUA DF, 2017).

A TAPP consiste na inserção de três portais - assim como a TEP - localizados na área umbilical e nos flancos direito e esquerdo. O portal umbilical deve ser inserido primeiro, seguido por insuflação do abdome e posteriormente, a introdução dos outros portais. Após a identificação dos ligamentos umbilicais mediais, bexiga, vasos epigástricos inferiores, canal deferente, cordão espermático, vasos ilíacos e defeitos da hérnia, deve ser realizada a incisão peritoneal na lateral do ligamento umbilical medial, acima do defeito da hérnia. $O$ estabelecimento do pneumoperitônio para acesso à cavidade abdominal apresenta grande risco de lesão visceral, devendo ser realizado cuidadosamente (SAROSI GA e BEN-DAVID K, 2015; HAZBÓN HR e LÓPEZATEHORTUA DF, 2017).

O retalho peritoneal deve ser desenvolvido no plano avascular, entre o peritônio e a fáscia transversal e deve ser mobilizado para expor a sínfise púbica, o ligamento de Cooper, o trato iliopúbico, estruturas do cordão, vasos epigástricos inferiores e espaços da hérnia (SAROSI GA e BEN-DAVID K, 2015; BITTNER R, el al., 2011).

A redução da hérnia direta da gordura pré-peritoneal é realizada por tração, enquanto a indireta deve ser mobilizada a partir de estruturas do cordão e reduzida para a cavidade peritoneal. Após a redução, a tela deve ser posicionada e, a depender do cirurgião, fixada. Por fim, o retalho peritoneal inferior desenvolvido durante o procedimento deve ser posicionado sobre a tela para isolá-la da cavidade peritoneal, utilizando sutura contínua (SAROSI GA e BEN-DAVID K, 2015; BITTNER R, et al., 2011).

\section{Vantagens e Desvantagens}

As técnicas laparoscópicas trouxeram vantagens em comparação com a técnica aberta, incluindo recuperação mais precoce, melhora da qualidade de vida, redução da dor pós-operatória e de morbidades (AGHAYEVA A, et al., 2020). A avaliação da qualidade de vida dos pacientes, a partir de diferentes instrumentos, tornou-se a principal forma de mensuração dos parâmetros do sucesso terapêutico (BANSAL VK, et al., 2017). Na pesquisa de Bansal VK, et al. (2013), os grupos com a técnica TEP e TAPP apresentaram melhora significativa na qualidade de vida no intervalo de 3 meses após o tratamento. O grupo TAPP apresentou melhora, com exceção no domínio de vitalidade e funções sociais, enquanto o TEP mostrou melhora em todos os domínios.

A função sexual é uma preocupação dos pacientes submetidos à cirurgia, sendo comum a queixa de seu prejuízo, portanto, é outro tópico abordado em vantagens das novas técnicas (BANSAL VK et al., 2017). A etiologia da disfunção testicular é ainda desconhecida, contudo, entende-se que pode ser pelo manuseio dos vasos testiculares e estruturas do cordão durante a dissecção, principalmente em cirurgias abertas (BANSAL VK, et al., 2017). 
Dessa forma, os estudos de Asuri K, et al. (2021) e de Bansal VK, et al. (2017) constataram melhora significativa na função sexual após técnicas TEP e TAPP em termos de volume testicular e manutenção dos níveis hormonais. Não houve diferença significativa entre ambas as técnicas. Essa melhora é associada à manipulação mínima de tecido na laparoscopia e menor resposta fibrótica, além da redução da dor no pósoperatório (BANSAL VK, et al., 2017).

Portanto, ambas as técnicas são formas de tratamentos aceitáveis e efetivas para reparo de hérnia inguinal e potenciais efeitos adversos são raros nas técnicas TAPP e TEP. Todavia, existem vantagens e desvantagens particulares de cada uma delas (BITTNER R, et al., 2015).

Quanto às vantagens da TEP, por não haver violação da cavidade peritoneal, há uma redução da dor pósoperatória em 3 meses, sobretudo quando é o primeiro reparo de hérnia inguinal. Além disso, ocorre menor tempo de internação hospitalar (CHEN L, et al., 2019; BANSAL VK, et al., 2013; AGHAYEVA A, et al., 2020). Contudo, o método TEP é um procedimento com longa curva de aprendizado e requer que o cirurgião esteja familiarizado com a anatomia, além do restrito espaço de abordagem (BANSAL VK, et al., 2013; AGHAYEVA $A$, et al., 2020). Ademais, há um risco aumentado de lesões vasculares durante a entrada extraperitoneal e dissecção, além de maior taxa de complicações cirúrgicas pós-operatórias em comparação com a TAPP (AGHAYEVA A, et al., 2020; BITTNER R, et al., 2015).

A TAPP tem a facilidade do aprendizado por não haver restrições de espaço (BANSAL VK, et al., 2013; AGHAYEVA A, et al., 2020). Por outro lado, por ter a cavidade peritoneal aberta, existe a possibilidade de aderências intestinais à tela, além de um risco aumentado de lesões viscerais durante 0 acesso transabdominal (AGHAYEVA A, et al., 2020). Ademais, a TAPP tem uma permanência hospitalar mais longa em comparação com TEP. (BITTNER R, et al., 2015).

\section{Complicações}

De acordo com o Consenso sobre as Diretrizes Internacionais para o Tratamento de Hérnias Inguinais, as técnicas laparoscópicas são recomendadas para o tratamento eletivo dessa condição (VAN VEENENDAAL $\mathrm{N}$, et al., 2020). Contudo, apesar de seus pontos benéficos, ambas as abordagens possuem complicações, algumas compartilhadas entre elas e outras mais comumente apresentadas em uma das técnicas específicas (VAN VEENENDAAL N, et al., 2020).

Dentre as complicações apresentadas nos estudos, evidenciou-se seromas, hematomas, infecções de sítio cirúrgico, lesões de órgãos abdominais e retenção urinária (ŁOMNICKI J, et al., 2018). Além disso, observou-se, em complicações mais tardias, o desenvolvimento de dor crônica e a necessidade de reabordagem cirúrgica devido à recorrência da hérnia. O risco total de complicações após o reparo das hérnias inguinais é de 15 a $28 \%$, sendo que as complicações que podem gerar mortalidade são mínimas, não sendo relatadas em nenhum dos trabalhos analisados (ŁOMNICKI J, et al., 2018; TORRES OC, et al., 2016).

\section{Seromas}

Dentre as complicações de curto prazo, o seroma está entre as mais citadas. Essa complicação é caracterizada pelo acúmulo de líquido na região submetida ao procedimento cirúrgico, sendo mais comum nos pacientes submetidos a TEP quando comparados à TAPP. Nesse mesmo estudo, foi relatado que após 7 dias da cirurgia 32,5\% dos pacientes submetidos a TEP apresentavam a complicação e, após 1 mês, 3,7\% ainda mantinham o quadro (BANSAL VK, et al., 2013).

Corroborando com esse resultado, Hung TY, et al. (2020) apresentou uma maior incidência dessa complicação com a TEP quando comparada a TAPP, assim como Varcus F et al. (2016), que identificou uma incidência de 2,27\% de seroma da região inguinal enquanto na TAPP não foi identificado qualquer caso. Todavia, Tulin A, et al. (2019) relatou uma incidência que se contrapõem ao padrão apresentado acima, sendo de 4 a 9,3\% em pacientes submetidos a TEP e 11,6\% aos submetidos a TAPP. Visando a redução dessa complicação, foi relatado que as taxas de seroma podem ser reduzidas na TEP com a implementação do uso de drenos (TULIN A, et al., 2019). 


\section{Dor pós-operatória e crônica}

A dor pode ser caracterizada como complicação dos procedimentos em dois casos, como dor no pósoperatório e como dor crônica. A dor pós-operatória, mensurada por meio de escores de dor e do requerimento de analgesia pelos pacientes, apresenta menor incidência nos operados pela TEP. Esse fato se deve a uma menor manipulação do peritônio parietal durante o procedimento laparoscópico (CHEN L, et al., 2019; BANSAL VK, et al., 2013; TORRES OC, et al., 2016). Quando foi analisado o consumo de analgésicos em casos de recorrência, não houve uma divergência entre ambas as técnicas (CHEN L, et al., 2019).

A dor crônica é caracterizada quando persiste por mais de 3 meses após a cirurgia. É normalmente referida como de baixa intensidade e difusa, porém contínua na região inguinal. Não houve uma diferença significativa entre as técnicas, sendo a incidência de 1,25\% para a TEP e 1,29\% para a TAPP (BANSAL VK, et al., 2013). Todavia, na análise realizada por Tulin A, et al. (2019), a incidência foi de 2,3\% na TEP e 4,6\% na TAPP.

Além disso, observou-se uma correlação entre fatores pré-operatórios (dor pré-operatória, dor nas primeiras 24h após o procedimento e ocupação com alto ou moderado esforço físico) e o surgimento da dor crônica. É importante ressaltar o impacto dessa complicação na morbidade e qualidade de vida dos pacientes, devendo ser ao máximo prevenida (BANSAL VK, et al., 2013).

\section{Infecções de sítio}

As infecções de sítio são caracterizadas como uma complicação local e, dentre os estudos analisados, não houve diferença significativa entre suas incidências e as técnicas abordadas. As infecções que ocorreram foram submetidas a tratamento com antibióticos e curativos, não sendo necessária a troca da tela usada no procedimento (BANSAL VK, et al., 2013; TORRES OC, et al., 2016; KÖCKERLING F, et al., 2015).

\section{Lesões viscerais}

Dentre as possíveis complicações por acometimento de órgãos temos como principais lesões as de bexiga e de intestino (VARCUS F, et al., 2016; KÖCKERLING F, et al., 2015). A lesão na bexiga apresentou uma incidência de 2,27\% na TEP (representando um caso), enquanto nenhuma ocorreu na TAPP. Já a lesão intestinal, ocorreu na região do cólon sigmóide pela TAPP e teve um acometimento superficial, atingindo apenas a camada serosa (VARCUS F, et al., 2016).

\section{Recorrência}

A taxa de recorrência das hérnias inguinais logo após a laparoscopia varia de 0 a 5,6\% para TEP, havendo redução dessa incidência conforme a evolução pós operatória. Já para a TAPP, a recidiva varia de 0,3 a 2,3\%, podendo se igualar com a da TEP (TORRES OC, et al., 2016; TULIN A, et al., 2019; SHI YH, et al., 2020; GASS M, et al., 2016). Dentre os fatores associados à essa recorrência estão a dissecção inadequada, cobertura insuficiente dos defeitos da parede, fixação inadequada e inexperiência do cirurgião (KÖCKERLING F, et al., 2015).

\section{Outras complicações}

Outras complicações que podem ser encontradas são: orquite, inguinodinia e hematomas (KÖCKERLING F, et al., 2015). O risco de lesão vascular, mais comumente nas artérias epigástricas inferiores, é mais presente na TEP devido ao espaço mais limitado dessa abordagem (SAROSI GA e BEN-DAVID K, 2015). Além disso, pode-se apresentar o edema de cordão, que ocorre como uma complicação recente de maior prevalência na TAPP $(29,6 \%)$ quando comparado a TEP $(12,6 \%)$ e o enfisema subcutâneo, presente na TEP com incidência de 6,8\% e na TAP de 2,17\% (VARCUS F, et al., 2016; BANSAL VK, et al., 2013).

A perda de sangue como complicação intraoperatória foi levantada por Sharma D, et al. (2015) e ficou evidenciado que essa perda em ambos os grupos se deu de forma mínima, caracterizando uma quantidade inferior ou igual a $50 \mathrm{ml}$ (SHARMA D, et al., 2015).

É importante ressaltar que as diferenças apresentadas entre as complicações das técnicas TAPP e TEP para o tratamento de hérnias inguinais representam mínimas divergências entre os dois métodos. Dessa forma, ambas as técnicas apresentam resultados comparáveis bastante semelhantes (BITTNER R e SCHWARZ J, 2019). 


\section{CONSIDERAÇÕES FINAIS}

Tendo em vista que a correção da hérnia inguinal é o procedimento cirúrgico mais realizado no mundo e que sua abordagem laparoscópica é cada vez mais utilizada, é importante saber as diferenças entre as técnicas laparoscópicas, TAPP e TEP, para se escolher com mais segurança qual utilizar. A atual revisão analisou os 29 estudos mais relevantes sobre o assunto. A partir dessa análise, foi demonstrado que não há divergência relevante na incidência de complicações pós-operatórias, na recorrência de reabordagem e no impacto na qualidade de vida dos pacientes que se submetem a uma das técnicas laparoscópicas. Concluise, portanto, que a escolha da melhor técnica deverá depender da familiaridade e das preferências do cirurgião e do serviço, assim como das características específicas de cada paciente.

\section{REFERÊNCIAS}

1. AGHAYEVA A, et al. Laparoscopic totally extraperitoneal vs robotic transabdominal preperitoneal inguinal hernia repair: Assessment of short- and long- term outcomes. The International Journal of Medical Robotics and Computer Assisted Surgery, 2020; 16(4): e2111.

2. AIOLFI A, et al. Primary inguinal hernia: systematic review and Bayesian network meta-analysis comparing open, laparoscopic transabdominal preperitoneal, totally extraperitoneal, and robotic preperitoneal repair. Hernia, 2019; $23(3)$ : 473-484.

3. ARRUDA WMQ, et al. Comparação entre as técnicas laparoscópica totalmente extraperitoneal (TEP) e transabdominal préperitoneal (TAPP) na hernioplastia inguinal: síntese de evidências clínicas. International Journal of Health Management Review, 2019; 5(2): 1-10.

4. ASURI K, et al. A prospective randomized comparison of sexual function and semen analysis following laparoscopic totally extraperitoneal (TEP) and transabdominal pre-peritoneal (TAPP) inguinal hernia repair. Surgical Endoscopy, 2021; 35(6): 2936-2941.

5. BANSAL VK, et al. A prospective randomized comparison of testicular functions, sexual functions and quality of life following laparoscopic totally extra-peritoneal (TEP) and trans-abdominal pre-peritoneal (TAPP) inguinal hernia repairs. Surgical endoscopy, 2017; 31(3): 1478-1486.

6. BITTNER R, et al. Guidelines for laparoscopic (TAPP) and endoscopic (TEP) treatment of inguinal hernia [International Endohernia Society (IEHS)]. Surgical endoscopy, 2011; 25(9): 2773-2843.

7. BITTNER R, et al. Update of guidelines on laparoscopic (TAPP) and endoscopic (TEP) treatment of inguinal hernia (International Endohernia Society). Surgical endoscopy, 2015; 29(2): 289-321.

8. BITTNER R, SCHWARZ J. Inguinal hernia repair: current surgical techniques. Langenbeck's archives of surgery, 2012; 397(2): 271-282.

9. BITTNER R, SCHWARZ J. Primary unilateral not complicated inguinal hernia: our choice of TAPP, why, results and review of literature. Hernia, 2019; 23(3): 417-428.

10. BROOKS DC. Overview of treatment for inguinal and femoral hernia in adults. UpToDate, Rosen M ed. UpToDate. Waltham, MA: UpToDate, 2020.

11. CHEN L, et al. Effects of transabdominal preperitoneal and totally extraperitoneal inguinal hernia repair: an update systematic review and meta-analysis of randomized controlled trials. Surgical endoscopy, 2019; 33(2): 418-428.

12. GASS M, et al. TAPP or TEP for recurrent inguinal hernia? Population-based analysis of prospective data on 1309 patients undergoing endoscopic repair for recurrent inguinal hernia. World journal of surgery, 2016;40(10): 2348-2352.

13. HAZBÓN HR, LÓPEZ-ATEHORTUA DF. Experience and results of laparoscopic inguinal herniorrhaphy. Revista de la Facultad de Medicina, 2017; 65(3): 429-432.

14. HUNG TY, et al. Safety of two common laparoscopic inguinal herniorrhaphy approaches: an updated systematic review with meta-analysis of randomized clinical trials. Translational Andrology and Urology, 2020; 9(5): 2007.

15. IUAMOTO LR, et al. Laparoscopic totally extraperitoneal (TEP) hernioplasty using two trocars: anatomical landmarks and surgical technique. ABCD. Arquivos Brasileiros de Cirurgia Digestiva (São Paulo), 2015; 28(2): $121-123$.

16. KÖCKERLING F, et al. TEP versus TAPP: comparison of the perioperative outcome in 17,587 patients with a primary unilateral inguinal hernia. Surgical endoscopy, 2015; 29(12): 3750-3760

17. ŁOMNICKI J, et al. Current treatment of the inguinal hernia: the role of the totally extraperitoneal (TEP) hernia repair. Folia Medica Cracoviensia, 2018; 58(3): 103-114.

18. LYU Y, et al. Comparison of endoscopic surgery and Lichtenstein repair for treatment of inguinal hernias: A network metaanalysis. Medicine, 2020; 99(6): e19134.

19. PATEL V, et al. Laparoscopic Inguinal Hernia Repair: Transabdominal Preperitoneal or Totally Extraperitoneal? Results of a 14-year Prospective Study. Chirurgia (Bucharest, Romania: 1990), 2020; 115(5): 600-608.

20. SAROSI GA e BEN-DAVID K. Laparoscopic inguinal and femoral hernia repair in adults. UptoDate, UptoDate, Waltham, MA, 2015.

21. SHARMA D, et al. Prospective randomized trial comparing laparoscopic transabdominal preperitoneal (TAPP) and laparoscopic totally extra peritoneal (TEP) approach for bilateral inguinal hernias. International Journal of Surgery, $2015 ; 22$ : 110-117.

22. SHI YH, et al. Comparison of the effect of mesh-plug, Lichtenstein, transabdominal preperitoneal, and totally extraperitoneal hernia repair: A network meta-analysis. Revista da Associação Médica Brasileira, 2020; 66(5): 687-691.

23. TORRES OC, et al. Experiencia Inicial En El Tratamiento Laparoscópico De La Hernia Inguinal En La II Cátedra De Clínica Quirúrgica, Hospital de Clínicas FCM-UNA. Cir. Parag., 2016; 40(2): 21-23.

24. TULIN A, et al. TAAP vs. TEP in inguinal hernia repair what is the evidence? A single center experience. Chirurgia (Bucur), 2019; 114(1): 67-72.

25. VACCA VM Jr. Inguinal hernia: A battle of the bulge. Nursing, 2017; 47(8): 28-35.

26. VAN VEENENDAAL N, et al. Consensus on international guidelines for management of groin hernias. Surgical endoscopy, 2020; 34(6): 2359-2377.

27. VARCUS F, et al. Laparoscopic repair of inguinal hernia TEP versus TAPP. Chirurgia (Bucur), 2016; 111(4): 308-312.

28. WEI FX, et al. Transabdominal preperitoneal (TAPP) versus totally extraperitoneal (TEP) for laparoscopic hernia repair: a meta-analysis. Surgical laparoscopy, endoscopy \& percutaneous techniques, 2015; 25(5): 375-383. 